\title{
DOUADY-EARLE EXTENSION OF THE STRONGLY SYMMETRIC HOMEOMORPHISM
}

\author{
YAN WU AND YI QI
}

\begin{abstract}
It is shown that the complex dilatation of the Douady-Earle extension of a strongly symmetric homeomorphism induces a vanishing Carleson measure on the unit disk $\mathbf{D}$. As application, it is proved that the VMO-Teichmüller space is a subgroup of the universal Teichmüller space.
\end{abstract}

\section{§1. Introduction}

Let $\mathbf{D}=\{z:|z|<1\}$ be the unit disk of the extended complex plane $\hat{\mathbf{C}}$ and let $\mathbf{D}^{*}=\hat{\mathbf{C}} \backslash \overline{\mathbf{D}}$ be the exterior of $\mathbf{D}$ and $S^{1}=\partial \mathbf{D}=\partial \mathbf{D}^{*}$ be the unit circle.

A sense-preserving homeomorphism $h: S^{1} \rightarrow S^{1}$ is said to be quasisymmetric if there exists some constant $M>0$ such that

$$
\frac{1}{M} \leq \frac{\left|h\left(I_{1}\right)\right|}{\left|h\left(I_{2}\right)\right|} \leq M
$$

for all pairs of adjacent arcs $I_{1}$ and $I_{2}$ on $S^{1}$ with the same arc-length $\left|I_{1}\right|=$ $\left|I_{2}\right|(\leq \pi)$. It is well known in [4] that a sense-preserving self-homeomorphism $h$ is quasisymmetric if and only if there exists some quasiconformal homeomorphism of $\mathbf{D}$ onto itself which has boundary values $h$.

Let $\mathrm{QS}\left(S^{1}\right)$ be the set of all quasisymmetric homeomorphisms of the unit circle $S^{1}$. Then $\mathrm{QS}\left(S^{1}\right)$ is a group under the composition of homeomorphisms. The universal Teichmüller space $T$ is defined as

$$
T=\mathrm{QS}\left(S^{1}\right) / \operatorname{Möb}\left(S^{1}\right),
$$

where $\operatorname{Möb}\left(S^{1}\right)$ is the group of Möbius transformations of $S^{1}$. It is well known that the universal Teichmüller space plays a significant role in the study of Teichmüller theory. For more details we refer to the books $[12,13,16,18]$.

2010 Mathematics Subject Classification. Primary 30F60; Secondary 32G15.

Key words and phrases. Douady-Earle extension; strongly symmetric homeomorphism; VMOTeichmüller space; Carleson measure.

The research is partially supported by the National Natural Science Foundation of China (Grant No. 11371045, 11301248).

Received July 9, 2015; revised October 13, 2015. 
For every $h \in \mathrm{QS}\left(S^{1}\right)$, it is proved in [9] that there exists a quasiconformal extension of $h$ to the unit disk, called the Douady-Earle extension, which is conformally invariant, that is,

$$
E(\alpha \circ h \circ \beta)=\alpha \circ E(h) \circ \beta
$$

holds for any $\alpha, \beta \in \operatorname{Möb}\left(S^{1}\right)$. Douady-Earle extension is very important in Teichmüller theory, which provides a great convenience to discuss Teichmüller spaces of Riemann surfaces on the unit disk, for instance.

A quasisymmetric homeomorphism $h$ of $S^{1}$ is called integrably asymptotic affine [7] if it admits a quasiconformal extension into $\mathbf{D}$ such that its complex dilatation $\mu$ is square integrable in the Poincare metric on $\mathbf{D}$, that is

$$
\iint_{\mathbf{D}} \frac{|\mu(z)|^{2}}{\left(1-|z|^{2}\right)^{2}} d x d y<\infty .
$$

It is proved in [7] that the complex dilatation of the Douady-Earle extension of an integrably asymptotic affine homeomorphism $h$ is square integrable in the Poincaré metric on $\mathbf{D}$.

An asymptotically conformal mapping $f$ of $\mathbf{D}$ is a quasiconformal homeomorphism of $\mathbf{D}$ with complex dilatation $\mu$ satisfying

$$
\lim _{|z| \rightarrow 1^{-}}|\mu(z)|=0 \text {. }
$$

A quasisymmetric homeomorphism $h$ of $S^{1}$ is called symmetric if it admits an asymptotically conformal extension on D. It is proved in [11] that the DouadyEarle extension of a symmetric homeomorphism is asymptotically conformal.

A quasisymmetric homeomorphism $h$ of $S^{1}$ is said to be strongly quasisymmetric if for each $\epsilon>0$, there exists a $\delta>0$ such that

$$
|E| \leq \delta|I| \Rightarrow|h(E)| \leq \epsilon|h(I)|
$$

where $I \subset S^{1}$ is an interval and $E \subset I$ is a measurable subset. It is equivalent to that [3] $h$ admits a quasiconformal extension into $\mathbf{D}$ which complex dilatation $\mu$ induces a Carleson measure $|\mu(z)|^{2} /\left(1-|z|^{2}\right) d x d y$ on D. It is shown in [8] that the complex dilatation of the Douady-Earle extension of a strongly quasisymmetric homeomorphism induces a Carleson measure. Furthermore, $h$ is strongly quasisymmetric if and only if $h$ is absolutely continuous and $\log h^{\prime} \in \operatorname{BMO}\left(S^{1}\right)$, the space of integrable functions on $S^{1}$ of bounded mean oscillation (see [6, 10, $14,20])$.

A quasisymmetric homeomorphism $h$ of $S^{1}$ is called strongly symmetric if $h$ is absolutely continuous and $\log h^{\prime} \in \operatorname{VMO}\left(S^{1}\right)$, the space of integrable functions on $S^{1}$ of vanishing mean oscillation (see $\left.[14,20,21]\right)$. The BMO-Teichmüller space and VMO-Teichmüller space are defined as the following models

$$
T_{b}=\operatorname{SQS}\left(S^{1}\right) / \operatorname{Möb}\left(S^{1}\right) \quad \text { and } \quad T_{v}=\operatorname{SS}\left(S^{1}\right) / \operatorname{Möb}\left(S^{1}\right),
$$

where $\operatorname{SQS}\left(S^{1}\right)$ and $\operatorname{SS}\left(S^{1}\right)$ are the sets of all strongly quasisymmetric and all strongly symmetric homeomorphisms of the unit circle $S^{1}$ respectively. The 
BMO-Teichmüller space and VMO-Teichmüller space are two important subspaces of the universal Teichmüller space which are fully studied $[1,3,5$, $8,23]$.

The purpose of this paper is to study the Douady-Earle extensions of strongly symmetric homeomorphisms. It is obtained that $h$ is a strongly symmetric homeomorphism if and only if $h$ admits a quasiconformal extension into $\mathbf{D}$ which complex dilatation $\mu$ induces a vanishing Carleson measure $|\mu(z)|^{2} /\left(1-|z|^{2}\right) d x d y$ on $\mathbf{D}$. Moreover, it is proved that the complex dilatation of the Douady-Earle extension of $h$ properly induces this vanishing Carleson measure. As application, it is gotten that the VMO-Teichmüller space $T_{v}$ is a subgroup of the universal Teichmüller space $T$.

\section{§2. Preliminaries}

In this section, we recall some notions and basic results on BMO-functions, $A_{\infty}$ weight functions and Carleson measures which will be needed in this paper. For more details we refer to $[6,10,14]$.

$\operatorname{BMO}\left(S^{1}\right)$ is the space of all integrable functions on $S^{1}$ of bounded mean oscillation (see $[6,10,14,20])$. An integrable function $u \in L^{1}\left(S^{1}\right)$ is said to be of bounded mean oscillation if

$$
\|u\|_{\mathrm{BMO}}=\sup _{I} \frac{1}{|I|} \int_{I}\left|u-u_{I}\right| d \theta<\infty,
$$

where $I$ is any arc on $S^{1},|I|$ is the length of $I$ and $u_{I}=\frac{1}{|I|} \int_{I} u d \theta$ is the average of $u$ over $I . \quad \operatorname{VMO}\left(S^{1}\right)$ is the subspace of $\operatorname{BMO}\left(S^{1}\right)$ which consists of all vanishing mean oscillation functions. A function $u \in \operatorname{BMO}\left(S^{1}\right)$ is said to be of vanishing mean oscillation if

$$
\lim _{|I| \rightarrow 0} \frac{1}{|I|} \int_{I}\left|u-u_{I}\right| d \theta=0 .
$$

Let $\mu=\omega(x) d x$ be a positive Borel measure on $\mathbf{R}$, finite on compact sets. $\omega(x)$ is called an $A_{\infty}$ weight function [14], denoted by $\omega \in A_{\infty}$, if

$$
\mu(E) / \mu(I) \leq C(|E| /|I|)^{\alpha}
$$

holds for any interval $I$ and any Borel subset $E$ of $I$, where $C>0$ and $\alpha>0$ are constants independent of $E$ and $I$. Let $h \in \mathrm{SS}\left(S^{1}\right)$, then $h$ is strongly quasisymmetric, and consequently $h^{\prime} \in A_{\infty}$ (see [14]).

For every $\omega \in A_{\infty}$, it holds the reverse Hölder inequality [6]. So there exists a constant $c>0$ and $p>1$ such that

$$
\frac{1}{|I|} \int_{I} \omega^{p}(x) d x \leq c\left(\frac{1}{|I|} \int_{I} \omega(x) d x\right)^{p} .
$$

for every interval $I$ in $\mathbf{R}$. 
The Carleson sector $S(I)$, based on $I$, is defined by

$$
S(I)=\left\{z=r e^{i \theta}: 1-\frac{|I|}{2 \pi} \leq r<1, e^{i \theta} \in I\right\} .
$$

A positive Borel measure $\lambda$ on $\mathbf{D}$ is called a bounded Carleson measure if there exists a positive constant $C$ such that

$$
\lambda(S(I)) \leq C|I|
$$

We say that $\lambda$ is a vanishing Carleson measure if

$$
\lambda(S(I))=o(|I|), \quad|I| \rightarrow 0 .
$$

For a positive measure $\lambda$ on $\mathbf{D}^{*}$, replacing $S(I)$ in the above definition by the following Carleson sector:

$$
S^{*}(I)=\left\{z=r e^{i \theta}: 1<r \leq 1+\frac{|I|}{2 \pi}, e^{i \theta} \in I\right\},
$$

We similarly obtain the definition of a bounded or vanishing Carleson measure on $\mathbf{D}^{*}$. Denote by $C M(\Omega)$ and $C M_{0}(\Omega)$ the set of all bounded Carleson measures and vanishing Carleson measures on $\Omega$, respectively.

We need a lemma in [23] for Carleson measure.

Lemma 2.1. For a positive measure $\lambda$ on $\mathbf{D}$, set

$$
\tilde{\lambda}(z)=\iint_{\mathbf{D}} \frac{\left(1-|z|^{2}\right)\left(1-|w|^{2}\right)}{|1-\bar{w} z|^{4}} \lambda(w) d u d v
$$

Then $\tilde{\lambda}$ is a bounded or vanishing Carleson measure if $\lambda$ is a bounded or vanishing Carleson measure on $\mathbf{D}$.

The Douady-Earle extension $w=E(h)(z)$ is defined by the equation

$$
F(z, w)=\frac{1}{2 \pi} \int_{S^{1}} \frac{h(t)-w}{1-\bar{w} h(t)} \frac{1-|z|^{2}}{|z-t|^{2}}|d t|=0 .
$$

For $h \in \mathrm{QS}\left(S^{1}\right)$, let $v(h)$ denote the Beltrami coefficient of the inverse mapping of the Douady-Earle extension $E(h)$, and $v$ denote the Beltrami coefficient of a quasiconformal extension of $h^{-1}$. Then we have the following result (for details, see $[15])$.

Lemma 2.2. There exists a constant $C(h)$ such that $\forall w \in \mathbf{D}$

$$
\frac{|v(h)(w)|^{2}}{1-|v(h)(w)|^{2}} \leq C(h) \iint_{\mathrm{D}} \frac{|v(\zeta)|^{2}}{1-|v(\zeta)|^{2}} \frac{\left(1-|w|^{2}\right)^{2}}{|1-\bar{\zeta} w|^{4}} d \xi d \eta
$$




\section{§3. Douady-Earle extension of a strongly symmetric homeomorphism}

Recall that for any $h \in \mathrm{QS}\left(S^{1}\right)$, there exists a unique pair of conformal mappings $f: \mathbf{D} \rightarrow f(\mathbf{D})$ and $g: \mathbf{D}^{*} \rightarrow \hat{\mathbf{C}} \backslash \overline{f(\mathbf{D})}$, called the normalized decomposition of $h$, satisfying $f(0)=f^{\prime}(0)-1=0, g(\infty)=\infty$ and $h=f^{-1} \circ g$ on $S^{1}$, respectively. Furthermore, $f$ can be extended to a quasiconformal mapping in the whole plane with Beltami coefficient $\mu_{f}$. At the same time, $h$ is called the normalized conformal welding mapping of $f$. It is known that $h \in \operatorname{QS}\left(S^{1}\right)$ if and only if $h^{-1} \in \mathrm{QS}\left(S^{1}\right)$. For $h \in \mathrm{SS}\left(S^{1}\right)$, we have

Proposition 3.1. For any $h \in \mathrm{QS}\left(S^{1}\right), f, g$ are the above normalized decomposition of $h$. The following conditions are equivalent:

(1) $h \in \mathrm{SS}\left(S^{1}\right)$;

(2) $h^{-1} \in \mathrm{SS}\left(S^{1}\right)$;

(3) There exists a quasiconformal extension $\psi(z): \mathbf{D} \rightarrow \mathbf{D}$ of $h^{-1}$ whose Beltrami coefficient $\mu$ induces a vanishing Carleson measure $|\mu(z)|^{2} /\left(1-|z|^{2}\right) d x d y$ on $\mathbf{D}$.

Proof. It should be pointed out that $(1) \Leftrightarrow(2)$ is implied in [23]. For completeness, we give the proof here.

Suppose that $h \in \mathrm{SS}\left(S^{1}\right)$ and $h=f^{-1} \circ g$, where $f, g$ are the normalized decomposition of $h$. Then $\log f^{\prime} \in \operatorname{VMOA}(\mathbf{D})$, the space of analytic functions in $\mathbf{D}$ of vanishing mean oscillation (see Theorem 4.1 in [23]). It is known that $\log f^{\prime} \in \operatorname{VMOA}(\mathbf{D})$ if and only if the quasicircle $\Gamma=f\left(S^{1}\right)=g\left(S^{1}\right)$ is asymptotically smooth (see Section 7.5 in [20]). Furthermore, we have $h^{-1}=g^{-1} \circ f=$ $(r j \circ g \circ j)^{-1} \circ(r j \circ f \circ j)$, where $j(z)=\bar{z}^{-1}$ is the standard reflection of the unit circle $S^{1}$ and $r$ is a constant such that $r(j \circ g \circ j)^{\prime}(0)=1$. So $r j \circ g \circ j$, $r j \circ f \circ j$ are the normalized decomposition of $h^{-1}$. Since $\Gamma$ is asymptotically smooth, then $r j \circ g \circ j\left(S^{1}\right)=r j(\Gamma)$ is also asymptotically smooth. This means $h^{-1} \in \mathrm{SS}\left(S^{1}\right)$ and $(1) \Rightarrow(2)$. With similar discussion, (2) $\Rightarrow(1)$.

Now we show that $(1) \Leftrightarrow(3)$. It is known that $h \in \operatorname{SS}\left(S^{1}\right)$ if and only if $f$ can be extended to a quasiconformal mapping to the whole plane, denoted also by $f$, whose complex dilatation $\mu_{f}$ satisfying $\left|\mu_{f}(z)\right|^{2} /\left(|z|^{2}-1\right) d x d y \in$ $C M_{0}\left(\mathbf{D}^{*}\right)$ [23]. Defining $\varphi(z)=g^{-1} \circ f(z), z \in \mathrm{D}^{*}$, then $\varphi(z)$ is the quasiconformal extension of $h^{-1}$ to $\mathrm{D}^{*}$ with Beltrami coefficient $v(z)=\mu_{f}(z)$ and $|v(z)|^{2} /\left(|z|^{2}-1\right) d x d y \in C M_{0}\left(\mathbf{D}^{*}\right)$. By reflection, $h^{-1}$ may be extended to a quasiconformal mapping $\psi(z)$ to $\mathbf{D}$ whose Beltrami coefficient $\mu(z)$ satisfies

$$
\mu(z)=\overline{v\left(\frac{1}{\bar{z}}\right)} \frac{z^{2}}{\bar{z}^{2}}, \quad z \in \mathbf{D} .
$$

For any subarc $I \in S^{1}(|I| \leq \pi)$, let $2 I$ be the subarc of $S^{1}$ with the same center of $I,|2 I|=2|I|$ and $z \in S(I)$. Then, by simple calculation, we get

$$
\iint_{S(I)} \frac{|\mu(z)|^{2}}{1-|z|^{2}} d x d y=\iint_{S^{\prime}(I)} \frac{|v(w)|^{2}}{|w|^{2}-1} \frac{1}{|w|^{2}} d u d v \leq \iint_{S^{*}(2 I)} \frac{|v(w)|^{2}}{|w|^{2}-1} d u d v
$$


where $S^{\prime}(I)$ is the refection sector of $S(I), S^{*}(2 I) \subset \mathbf{D}^{*}$ is the Carleson sector over $2 I$ on $\mathbf{D}^{*}$ and $S^{\prime}(I) \subset S^{*}(2 I)$.

For any given $\varepsilon>0$, since $|v(w)|^{2} /\left(|w|^{2}-1\right) d u d v \in C M_{0}\left(\mathbf{D}^{*}\right)$, there exists a $\delta>0$ such that

$$
\iint_{S^{*}(2 I)} \frac{|v(w)|^{2}}{|w|^{2}-1} d u d v<2 \varepsilon|I|
$$

holds for every subarc $I \subset S^{1}$ with $|I| \leq \delta$. So $|\mu(z)|^{2} /\left(1-|z|^{2}\right) d x d y \in C M_{0}(\mathbf{D})$ and $(1) \Rightarrow(3)$.

Conversely, if condition (3) holds, by quasiconformal reflection, there exists a quasiconformal extension $\phi(z): \mathbf{D}^{*} \rightarrow \mathbf{D}^{*}$ of $h^{-1}$ with Beltrami coefficient $\mu_{\phi}(z)$ satisfying $\left|\mu_{\phi}(z)\right|^{2} /\left(|z|^{2}-1\right) d x d y \in C M_{0}\left(\mathbf{D}^{*}\right)$. Let $\tilde{f}=g \circ \phi$, it is easy to see that $\tilde{f}$ is the quasiconformal extension of $f$ and $\left|\mu_{\tilde{f}}(z)\right|^{2} /\left(|z|^{2}-1\right) d x d y \in$ $C M_{0}\left(\mathbf{D}^{*}\right)$. Thus $(3) \Rightarrow(1)$.

Now we prove that the complex dilatation of the Douady-Earle extension of a strongly symmetric homeomorphism induces a vanishing Carleson measure on $\mathbf{D}$.

THEOREM 3.1. If $h \in S S\left(S^{1}\right)$, that is, $h$ is a strongly symmetric homeomorphism on $S^{1}$. Let $\mu$ be the complex dilatation of the Douady-Earle extension $\Phi=E(h)$. Then it holds that $|\mu(z)|^{2} /\left(1-|z|^{2}\right) d x d y \in C M_{0}(\mathbf{D})$.

In order to prove Theorem 3.1, we need some preparations.

Set $\zeta_{k}=e^{2 k \pi i / 3}(k=1,2,3)$. For every $w \in \mathbf{D}$, let $\tau$ be the Möbius transformation of $\mathbf{D}$ onto itself with $\tau(0)=w$ and $\tau\left(\zeta_{2}\right)=w /|w|$. Denote $w_{k}=\tau\left(\zeta_{k}\right)$ $(k=1,2,3)$ and let $J_{w}$ be the subarc of $S^{1}$ with endpoints $w_{1}$ and $w_{3}$ and containing $w_{2}$. Then we have the following lemma.

LEMMA 3.1. Let $h$ be a symmetric homeomorphism of $S^{1}$ and $\Phi$ be the Douady-Earle extension of $h$, then there exist positive constants $C_{1}$ and $C_{2}$ depending only on $h$, such that

$$
\begin{gathered}
2(1-|w|) \leq\left|J_{w}\right| \leq 2 \pi(1-|w|) \\
\frac{1}{C_{1}} \frac{\left|h^{-1}\left(J_{w}\right)\right|}{\left|J_{w}\right|} \leq \frac{1-\left|\Phi^{-1}(w)\right|^{2}}{1-|w|^{2}} \leq C_{1} \frac{\left|h^{-1}\left(J_{w}\right)\right|}{\left|J_{w}\right|}
\end{gathered}
$$

and

$$
\frac{\left(1-|w|^{2}\right)^{2}}{\left(1-\left|\Phi^{-1}(w)\right|^{2}\right)^{2}} J_{\Phi^{-1}}(w) \leq C_{2} .
$$

Proof. Since $\Phi$ is the Douady-Earle extension of $h$, it is bi-Lipschitz with respect to the Poincaré metric and the Lipschitz constant $C=C(K)$ depends only on the maximal dilatation $K=K_{\Phi}$ of $\Phi$ [9]. Hence, $\Phi^{-1}$ is also bi-Lipschitz 
with respect to the Poincaré metric with the same Lipschitz constant $C=C(K)$. So,

$$
\frac{1}{C(K)} \rho(w)|d w| \leq \rho\left(\Phi^{-1}(w)\right)\left|d \Phi^{-1}(w)\right| \leq C(K) \rho(w)|d w|,
$$

which implies (3.3) with $C_{2}=C(K)^{2}$ directly.

Let $z_{k}=h^{-1}\left(w_{k}\right)(k=1,2,3)$ and $\sigma$ be the Möbius transformation of $\mathbf{D}$ onto itself with $\sigma\left(\zeta_{k}\right)=z_{k}(k=1,2,3)$. Set $\Phi^{*}=\tau^{-1} \circ \Phi \circ \sigma$. Then $\Phi^{*}$ is the Douady-Earle extension of the sense-preserving quasisymmetric $\left.\Phi^{*}\right|_{S^{1}}=\tau^{-1}$ 。 $h \circ \sigma$ and can be extended to a $K=K_{\Phi}$-quasiconformal mapping of $\mathbf{C}$ onto itself by reflection. Thus, $\left.\Phi^{*}\right|_{S^{1}}$ is $\eta_{K}$-quasisymmetric by Corollary 3.10.4 in [2], where

$$
\eta_{K}(t)=\lambda(K)^{2 K} \max \left\{t^{K}, t^{1 / K}\right\}, \quad t \in[0,+\infty)
$$

and

$$
\lambda(K)=\sup \left\{\left|f\left(e^{i \theta}\right)\right|: f: \mathbf{C} \rightarrow \mathbf{C} \text { is } K \text {-q.c. and fixes } 0,1,0 \leq \theta \leq 2 \pi\right\} .
$$

Therefore, by Proposition 5.21 in [20], there exists a constant $r^{\prime} \in(0,1)$ which depends only on $K$ but not on $w$, such that $\left|\Phi^{*}(0)\right| \leq r^{\prime}<1$.

As $\Phi^{*}$ is the Douady-Earle extension of the sense-preserving quasisymmetric $\left.\Phi^{*}\right|_{S^{1}}=\tau^{-1} \circ h \circ \sigma$, it is bi-Lipschitz with respect to the Poincaré metric, where the Lipschitz constant $C(K) \geq 1$ depends only on $K$ [9]. Thus,

$$
\log \frac{1+\left|\Phi^{*-1}(0)\right|}{1-\left|\Phi^{*-1}(0)\right|} \leq C(K) \log \frac{1+\left|\Phi^{*}(0)\right|}{1-\left|\Phi^{*}(0)\right|}
$$

This implies that

$$
\left|\Phi^{*-1}(0)\right| \leq r_{0}<1,
$$

where $r_{0}$ is a constant depending only on $K$ but not on the choice of $w$.

It is easy to see that $\tau(\zeta)=\left(\zeta+e^{i \alpha} w\right) /\left(e^{i \alpha}+\zeta \bar{w}\right)$, where $\alpha=\frac{4 \pi}{3}-\theta$ and $\theta$ is the argument of $w$. By a simple computation, we have

$$
\left|w_{1}-w_{2}\right|=\frac{\sqrt{3}(1-|w|)}{\left|\zeta_{1}+\right| w||}, \quad\left|w_{2}-w_{3}\right|=\frac{\sqrt{3}(1-|w|)}{\left|\zeta_{2}+\right| w||},
$$

and

$$
\left|w_{1}-w_{3}\right|=\frac{\sqrt{3}(1+|w|)(1-|w|)}{\left|\zeta_{2}+\right| w||\left|\zeta_{1}+\right| w||} .
$$

Consequently, it is gotten that $\left|w_{1}-w_{2}\right|=\left|w_{2}-w_{3}\right|$ and

$$
1-|w| \leq\left|w_{1}-w_{2}\right| \leq 2(1-|w|) \text {. }
$$

So, $\left|w_{1}-w_{2}\right|,\left|w_{2}-w_{3}\right|,\left|w_{1}-w_{3}\right|$ are all comparable with $1-|w|$ and the constants appeared in the comparisons are universal, and

$$
\left|J_{w}\right| \geq\left|w_{1}-w_{2}\right|+\left|w_{2}-w_{3}\right| \geq 2(1-|w|) .
$$


By Jordan inequality,

$$
\left|J_{w}\right|=2\left|\widehat{w_{1} w_{2}}\right| \leq \pi\left|w_{1}-w_{2}\right| \leq 2 \pi(1-|w|) .
$$

Thus, (3.1) is true.

We now prove that $\left|z_{1}-z_{2}\right|,\left|z_{2}-z_{3}\right|$ and $\left|z_{3}-z_{1}\right|$ are all comparable with $1-\left|\Phi^{-1}(w)\right|$ and the constants appeared in the comparisons depend only on $K=K_{\phi}$.

Let $z=\Phi^{-1}(w)$ and let $\zeta^{\prime} \in S^{1}$ such that $\sigma\left(\zeta^{\prime}\right)=z /|z|$. Set

$$
\sigma(\zeta)=e^{i \beta} \frac{\zeta-a}{1-\bar{a} \zeta}, \quad \zeta \in \mathbf{D},
$$

where $a \in \mathbf{D}$ and $\beta \in \mathbf{R}$ are constants determined by $\sigma$. Then

$$
\frac{\left|z_{i}-z_{j}\right|}{1-|z|}=\frac{\left|\sigma\left(\zeta_{i}\right)-\sigma\left(\zeta_{j}\right)\right|}{\left|\sigma\left(\zeta^{\prime}\right)-\sigma\left(\Phi^{*-1}(0)\right)\right|}=\frac{\left|\zeta_{i}-\zeta_{j}\right|}{\left|\zeta^{\prime}-\Phi^{*-1}(0)\right|} \frac{\left|1-\bar{a} \zeta^{\prime}\right|\left|1-\bar{a} \Phi^{*-1}(0)\right|}{\left|1-\bar{a} \zeta_{i}\right|\left|1-\bar{a} \zeta_{j}\right|}
$$

for $1 \leq i<j \leq 3$. If $\arg a \in[-\pi / 3, \pi / 3)$, then

$$
\left|1-\bar{a} \zeta_{1}\right| \geq \sqrt{3} / 2 \text { and }\left|1-\bar{a} \zeta_{2}\right| \geq \sqrt{3} / 2
$$

Thus, by (3.5) and (3.6),

$$
\frac{\left|z_{1}-z_{2}\right|}{1-|z|} \leq \frac{\sqrt{3}}{1-r_{0}} \cdot \frac{16}{3}
$$

Similarly, if $\arg a \in[\pi / 3, \pi)$ or $[\pi, 5 \pi / 3),(3.7)$ is also true for replacing $\left|z_{1}-z_{2}\right|$ by $\left|z_{1}-z_{3}\right|$ or $\left|z_{2}-z_{3}\right|$, respectively.

On the other hand,

$$
\frac{1-|z|}{\left|z_{i}-z_{j}\right|} \leq \frac{\left|z_{i}-z\right|}{\left|z_{i}-z_{j}\right|}=\frac{\left|\zeta_{i}-\Phi^{*-1}(0)\right|}{\left|\zeta_{i}-\zeta_{j}\right|} \frac{\left|1-\bar{a} \zeta_{j}\right|}{\left|1-\bar{a} \Phi^{*-1}(0)\right|} \leq \frac{4}{\sqrt{3}} \frac{1}{1-r_{0}}
$$

for $1 \leq i<j \leq 3$. Since $h$ is a symmetric homeomorphism and $\left|w_{1}-w_{2}\right|=$ $\left|w_{2}-w_{3}\right|$, then $\left|z_{1}-z_{2}\right|,\left|z_{2}-z_{3}\right|$ and $\left|z_{3}-z_{1}\right|$ can be compared with each other and the constants in the comparisons depend only on $K$. Thus, all these three quantities are all comparable with $1-|z|$ and constants in the comparisons depend only on $r_{0}=r_{0}(K)$ but independent on $w$.

Therefore, there exists a constant $C \geq 1$ depending only on $K$ such that

$$
\frac{1}{C} \frac{\left|h^{-1}\left(w_{1}\right)-h^{-1}\left(w_{3}\right)\right|}{\left|w_{1}-w_{3}\right|} \leq \frac{1-|z|}{1-|w|} \leq C \frac{\left|h^{-1}\left(w_{1}\right)-h^{-1}\left(w_{3}\right)\right|}{\left|w_{1}-w_{3}\right|},
$$

which implies (3.2) directly. The proof of Lemma 3.1 is completed.

Now we prove the Theorem 3.1.

Proof. For every $h \in \mathrm{SS}\left(S^{1}\right)$, by proposition 3.1, there exists a quasiconformal extension $g$ of $h^{-1}$ satisfying $\left|\mu_{g}(z)\right|^{2} /\left(1-|z|^{2}\right) d x d y \in C M_{0}(\mathbf{D})$. Let $v$ 
denote the Beltrami coefficient of the inverse mapping $\Phi^{-1}$ of the Douady-Earle extension $\Phi$. By Lemma 2.2, there exists a constant $C(h)$ such that $\forall w \in \mathbf{D}$

$$
\frac{|v(w)|^{2}}{1-|v(w)|^{2}} \leq C(h) \iint_{\mathrm{D}} \frac{\left|\mu_{g}(\zeta)\right|^{2}}{1-\left|\mu_{g}(\zeta)\right|^{2}} \frac{\left(1-|w|^{2}\right)^{2}}{|1-\bar{\zeta} w|^{4}} d \xi d \eta
$$

Furthermore,

$$
\begin{aligned}
\frac{|v(w)|^{2}}{1-|w|^{2}} & \leq C(h) \iint_{\mathrm{D}} \frac{1-|v(w)|^{2}}{1-\left|\mu_{g}(\zeta)\right|^{2}} \frac{\left|\mu_{g}(\zeta)\right|^{2}}{1-|\zeta|^{2}} \frac{\left(1-|w|^{2}\right)\left(1-|\zeta|^{2}\right)}{|1-\bar{\zeta} w|^{4}} d \xi d \eta \\
& \leq \frac{C(h)}{1-\left\|\mu_{g}\right\|_{\infty}^{2}} \iint_{\mathrm{D}} \frac{\left|\mu_{g}(\zeta)\right|^{2}}{1-|\zeta|^{2}} \frac{\left(1-|w|^{2}\right)\left(1-|\zeta|^{2}\right)}{|1-\bar{\zeta} w|^{4}} d \xi d \eta
\end{aligned}
$$

It follows from Lemma 2.1 that $|v(w)|^{2} /\left(1-|w|^{2}\right) d u d v \in C M_{0}(\mathbf{D})$. In what follows we prove that $|v(w)|^{2} /\left(1-|w|^{2}\right) d u d v \in C M_{0}(\mathbf{D})$ implies $|\mu(z)|^{2} /$ $\left(1-|z|^{2}\right) d x d y \in C M_{0}(\mathbf{D})$.

Since $h \in \mathrm{SS}\left(S^{1}\right), h$ is a symmetric homeomorphism [22], namely,

$$
\frac{\left|h\left(I_{1}\right)\right|}{\left|h\left(I_{2}\right)\right|}=1+o(1)
$$

holds for every pair of adjacent subarcs $I_{1}$ and $I_{2}$ in $[0,2 \pi]$ with $\left|I_{1}\right|=$ $\left|I_{2}\right| \rightarrow 0_{+}$.

For every $I \subset S^{1}$, set $I=I_{1}+I_{1}^{\prime}$ and $2 I=I_{2}+I_{1}+I_{1}^{\prime}+I_{2}^{\prime}$, where $I_{2}, I_{1}, I_{1}^{\prime}$, $I_{2}^{\prime}$ are adjacent subarcs with $\left|I_{1}\right|=\left|I_{1}^{\prime}\right|=\left|I_{2}\right|=\left|I_{2}^{\prime}\right|$. Then we have

$$
\left|h\left(I_{1}+I_{2}\right)\right|=2\left|h\left(I_{1}\right)\right|+o(1)=|h(I)|+o(1)
$$

and

$$
\left|h\left(I_{1}^{\prime}+I_{2}^{\prime}\right)\right|=2\left|h\left(I_{1}^{\prime}\right)\right|+o(1)=|h(I)|+o(1)
$$

as $|I| \rightarrow 0_{+}$. Thus,

$$
\frac{|h(2 I)|}{|h(I)|}=2+o(1), \quad|I| \rightarrow 0_{+} .
$$

Furthermore, for a positive integer $N>1$, it is not hard to verify that

$$
\frac{|h(N I)|}{|h(I)|}=N+o(1), \quad|I| \rightarrow 0_{+},
$$

where $I$ and $N I$ are the subarcs of $S^{1}$ with the same center and $|N I|=N|I|$.

Let $z_{0}$ be the center of $I$ and let $D(2 I)$ be the disk centered at $z_{0}$ and $D(2 I) \cap \partial \mathbf{D}=2 I$. It is easy to verify that the Carleson sector $S(I) \subset D(2 I)$ for every $I$ with $|I|<\pi$. By reflections and pre-compositing a Teichmüller shift [24] (A Teichmüller shift mapping on the unit disk $\mathbf{D}$ is the uniquely extremal 
mapping $T\left[w_{1}, w_{2}\right]$ which sends $w_{1}$ to $w_{2}$ and is equal to the identity on $\left.\partial \mathbf{D}\right)$, $\Phi$ can be extended to a $K^{\prime}$-quasiconformal mapping $\tilde{\Phi}$ of $\mathbf{C}$ onto itself with $\left.\tilde{\Phi}\right|_{\overline{\mathbf{D}}}=\Phi$, where $K^{\prime}$ depends only on $\Phi$. Since it is clear that

$$
\max _{w \in \partial \Phi(S(I)) \backslash \partial \mathbf{D}}\left|w-h\left(z_{0}\right)\right| \leq \max _{z \in \partial D(2 I)}\left|\tilde{\Phi}(z)-h\left(z_{0}\right)\right|
$$

and

$$
\min _{z \in \partial D(2 I)}\left|\tilde{\Phi}(z)-h\left(z_{0}\right)\right| \leq|h(2 I)|,
$$

so, by Teichmüller distortion theorem [17] and (3.8), we have

$$
\max _{w \in \partial \Phi(S(I))}\left|w-h\left(z_{0}\right)\right| \leq \lambda\left(K^{\prime}\right)|h(2 I)| \leq 3 \lambda\left(K^{\prime}\right)|h(I)|
$$

for sufficient small arc $I$, where $\lambda\left(K^{\prime}\right)$ is defined in (3.4) depending only on the maximal dilatation $K^{\prime}$. Choose an integer $N^{\prime}$ depending only on $K^{\prime}$ with $N^{\prime} \geq 6 \pi \lambda\left(K^{\prime}\right)$. Then by the definition of the Carleson sector, we have

$$
\Phi(S(I)) \subset S\left(N^{\prime} h(I)\right) .
$$

Denote $d \lambda=|\mu(z)|^{2} /\left(1-|z|^{2}\right) d x d y$ and $d \lambda^{\prime}=|v(w)|^{2} /\left(1-|w|^{2}\right) d u d v$. For any given $\varepsilon>0$, as we have just proved that $\lambda^{\prime}$ is a vanishing Carleson measure, there exists a $\delta^{\prime}>0$ such that

$$
\lambda^{\prime}(S(J))<\frac{\varepsilon}{4}|J|
$$

for every subarc $J \subset S^{1}$ with $|J| \leq \delta^{\prime} / 2$.

Let $J=N^{\prime} h(I)$ be the open subarc of the same center point with $h(I)$ and $|J|=N^{\prime}|h(I)|$. Then there is a $\delta_{1}>0$ such that $|J| \leq \delta^{\prime} / 2$ and $\Phi(S(I)) \subset S(J)$ holds for every subarc $I$ on $S^{1}$ with $|I|<\delta_{1}$.

By the properties of integral,

$$
\begin{aligned}
\lambda(S(I))=\iint_{S(I)} \frac{|\mu(z)|^{2}}{1-|z|^{2}} d x d y & =\iint_{\Phi(S(I))} \frac{1-|w|^{2}}{1-\left|\Phi^{-1}(w)\right|^{2}} J_{\Phi^{-1}}(w) d \lambda^{\prime} \\
& \leq \iint_{S(J)} \frac{1-|w|^{2}}{1-\left|\Phi^{-1}(w)\right|^{2}} J_{\Phi^{-1}}(w) d \lambda^{\prime}
\end{aligned}
$$

Then, from (3.2) and (3.3) in Lemma 3.1, we have

$$
\lambda(S(I)) \leq C \iint_{S(J)} \frac{\left|h^{-1}\left(J_{w}\right)\right|}{\left|J_{w}\right|} d \lambda^{\prime},
$$

where $C=C_{1} C_{2}$ is a constant depending only on $K$.

Let $\psi$ be a lift of $h^{-1}$ to the real line $\mathbf{R}$ over the obvious covering mapping. Then $\psi$ is strictly increasing, continuous and $\psi(\theta+2 \pi)-\psi(\theta)=2 \pi$. 
As $h^{-1} \in S S\left(S^{1}\right), \psi$ is differentiable almost everywhere in $\mathbf{R}$ and

$$
\left(h^{-1}\right)^{\prime}\left(e^{i \theta}\right)=e^{i(\psi(\theta)-\theta)} \psi^{\prime}(\theta) .
$$

Let $2 J$ be the arc on $S^{1}$ with the same center as $J$ and of length $2|J|$. Choose a component of the lift of $2 J$, which is an open interval, and denoted by $2 J$. Denote also by $J$ the component lift of $J$ contained in the component $2 J$ and $I$ the component lift of $I$ contained in $\psi(J)$. Let

$$
\phi(\theta)=\psi^{\prime}(\theta) \chi_{2 J}(\theta)
$$

where $\chi_{2 J}$ is the characteristic function of $2 J$ on $\mathbf{R}$. Let

$$
M \phi(\theta)=\sup _{\theta \in J^{\prime}} \frac{1}{\left|J^{\prime}\right|} \int_{J^{\prime}}|\phi(t)| d t
$$

be the Hardy-Littlewood maximal function of $\phi$, where the supremum is taken over all intervals $J^{\prime}$ containing $\theta$. Then

$$
M \phi(\theta) \geq\left|h^{-1}\left(J^{\prime}\right)\right| /\left|J^{\prime}\right|
$$

holds for all subarc $J^{\prime} \subset 2 J$ containing $\theta$.

By a property of Hardy-Littlewood maximal functions, $\{\theta \in \mathbf{R}: M \phi(\theta)>k\}$ is an open set for every $k>0$. Thus,

$$
\{\theta \in 2 J: M \phi(\theta)>k\}=2 J \cap\{\theta \in \mathbf{R}: M \phi(\theta)>k\}
$$

is open and consequently,

$$
\{\theta \in 2 J: M \phi(\theta)>k\}=\bigcup J_{l},
$$

where $\left\{J_{l}\right\}$ is a finite or infinite sequence of disjoint intervals contained in $J$.

We may assume that $|J|<\frac{\pi}{4}$. Let

$$
T\left(J_{l}\right)=\left\{w=r e^{i \theta}: 1-\frac{2\left|J_{l}\right|}{\pi} \leq r<1, e^{i \theta} \in J_{l}\right\} .
$$

Then,

$$
\left\{w \in S(J): \frac{\left|h^{-1}\left(J_{w}\right)\right|}{\left|J_{w}\right|}>k\right\} \subset \bigcup T\left(J_{l}\right) .
$$

Indeed, if $w \in S(J)$ and

$$
\frac{\left|h^{-1}\left(J_{w}\right)\right|}{\left|J_{w}\right|}>k
$$

then by the definition of Carleson sector, $1-|w|<|J| / 2 \pi$. So by (3.1) in Lemma 3.1, we have $\left|J_{w}\right|<|J|$ and consequently, $J_{w} \subset 2 J$. Thus, by (3.10) 
and (3.13), $e^{i \theta}:=w /|w| \in \bigcup J_{l}$. If $w \notin \bigcup T\left(J_{l}\right)$, then $\left|J_{l}\right|<\frac{\pi}{2}(1-|w|)$ for $J_{l}$ containing $w /|w|$. Thus, by (3.1), $\left|J_{w}\right|>\left|J_{l}\right|$. So, there exists a $e^{i \theta^{\prime}} \in J_{w} \backslash \bigcup J_{l}$ such that $M \phi\left(\theta^{\prime}\right)>k$. This contradicts to (3.11). Therefore, (3.12) holds.

Since $\left|J_{l}\right| \leq 2|J| \leq \delta^{\prime}$, then for the above $\varepsilon>0$,

$$
\begin{aligned}
\lambda^{\prime}\left(\left\{w \in S(J): \frac{\left|h^{-1}\left(J_{w}\right)\right|}{\left|J_{w}\right|}>k\right\}\right) & \leq \sum_{j} \lambda^{\prime}\left(T\left(J_{l}\right)\right) \leq \varepsilon \sum_{l}\left|J_{l}\right| \\
& =\varepsilon|\{\theta \in 2 J: M \phi(\theta)>k\}| .
\end{aligned}
$$

So, we have

$$
\iint_{S(J)} \frac{\left|h^{-1}\left(J_{w}\right)\right|}{\left|J_{w}\right|} d \lambda^{\prime} \leq \varepsilon \int_{2 J} M \phi d \theta .
$$

Since $\psi^{\prime}(\theta)$ belongs to the class of weights $A_{\infty}$, it holds the inverse hölder inequality (2.1) for some $p>1$ and $c>0$, that is,

$$
\frac{1}{2|J|} \int_{2 J} \psi^{\prime p} d \theta \leq c\left(\frac{1}{2|J|} \int_{2 J} \psi^{\prime} d \theta\right)^{p} .
$$

By Hölder inequality, for $q>1,1 / p+1 / q=1$, we have

$$
\int_{2 J} M \phi d \theta \leq(2|J|)^{1 / q}\left(\int_{2 J}(M \phi)^{p} d \theta\right)^{1 / p} .
$$

Furthermore, by Muckenhoupt theory (see §VI.6 of [14]), there exists a constant $C_{p}$ for $p>1$, independent of $\phi$, such that

$$
\int_{2 J}(M \phi)^{p} d \theta \leq \int_{\mathbf{R}}(M \phi)^{p} d \theta \leq C_{p} \int_{\mathbf{R}} \phi^{p} d \theta=C_{p} \int_{2 J} \psi^{\prime p} d \theta
$$

From (3.15)-(3.17), we have

$$
\int_{2 J} M \phi(\theta) d \theta \leq\left(c C_{p}\right)^{1 / p} \int_{2 J} \psi^{\prime}(\theta) d \theta
$$

Combining (3.9), (3.14) and (3.18), we get

$$
\lambda(S(I)) \leq C^{\prime} \varepsilon \int_{2 J} \psi^{\prime}(\theta) d \theta \leq C^{\prime} \varepsilon\left|h^{-1}(2 J)\right|
$$

for $|I|<\delta_{1}$, where $C^{\prime}=C\left(c C_{p}\right)^{1 / p}$ and $2 J=2 N^{\prime} h(I)$. By (3.8),

$$
\frac{\left|h^{-1}(2 J)\right|}{|I|}=2 N^{\prime}+o(1), \quad|I| \rightarrow 0_{+} .
$$


So for the above $\varepsilon>0$, there exists a positive number $\delta$ with $\delta<\delta_{1}$ such that

$$
\lambda(S(I)) \leq C^{\prime}\left(2 N^{\prime}+1\right) \varepsilon|I| .
$$

holds for every subarc $I$ on $S^{1}$ with $|I|<\delta$. Hence $|\mu(z)|^{2} /\left(1-|z|^{2}\right) d x d y \in$ $C M_{0}(\mathbf{D})$. The proof of this Theorem is completed.

\section{An application of Theorem 3.1}

As an application of Theorem 3.1, we prove the following theorem.

THEOREM 4.1. $T_{v}$ is a subgroup of $T$.

Proof. It is clear that the universal Teichmüller space $T$ and the VMOTeichmüller space $T_{v}$ can be identified as the spaces of all normalized quasisymmetric and all strongly symmetric homeomorphisms of $S^{1}$ respectively. Here, a homeomorphism of $S^{1}$ is called normalized if it fixes \pm 1 and $i$.

Let $h_{1}, h_{2} \in T_{v}$ be the normalized strongly symmetric homeomorphisms and $\Phi=E\left(h_{1}\right)$ be the Douady-Earle extension of $h_{1}$ with the Beltrami differential $\mu_{1}$. By Theorem 3.1, $\left|\mu_{1}(z)\right|^{2} /\left(1-|z|^{2}\right) d x d y \in C M_{0}(\mathbf{D})$. Furthermore, by Proposition 3.1, there exists a quasiconformal extension $f$ of $h_{2}$ with Beltrami differential $\mu_{2}$ satisfying $\left|\mu_{2}(z)\right|^{2} /\left(1-|z|^{2}\right) d x d y \in C M_{0}(\mathbf{D})$. Let $\rho$ be the Beltrami differential of $f \circ \Phi^{-1}$, then for any $z \in \mathbf{D}$,

$$
|\rho(\Phi(z))|^{2}=\left|\frac{\mu_{2}(z)-\mu_{1}(z)}{1-\mu_{2}(z) \overline{\mu_{1}(z)}}\right|^{2} \leq \frac{2\left(\left|\mu_{1}(z)\right|^{2}+\left|\mu_{2}(z)\right|^{2}\right)}{\left(1-\left\|\mu_{1}\right\|_{\infty}\left\|\mu_{2}\right\|_{\infty}\right)^{2}} .
$$

Thus,

$$
\begin{aligned}
\iint_{S(I)} \frac{|\rho(w)|^{2}}{1-|w|^{2}} d u d v= & \iint_{\Phi^{-1}(S(I))} \frac{|\rho(\Phi(z))|^{2}}{1-|\Phi(z)|^{2}} J_{\Phi}(z) d x d y \\
\leq & C \iint_{S(N J)} \frac{\left|\mu_{1}(z)\right|^{2}+\left|\mu_{2}(z)\right|^{2}}{1-|z|^{2}} \frac{1-|z|^{2}}{1-|\Phi(z)|^{2}} J_{\Phi}(z) d x d y \\
= & C \iint_{S(N J)} \frac{1-|z|^{2}}{1-|\Phi(z)|^{2}} J_{\Phi}(z) \cdot \frac{\left|\mu_{1}(z)\right|^{2}}{1-|z|^{2}} d x d y \\
& +C \iint_{S(N J)} \frac{1-|z|^{2}}{1-|\Phi(z)|^{2}} J_{\Phi}(z) \cdot \frac{\left|\mu_{2}(z)\right|^{2}}{1-|z|^{2}} d x d y
\end{aligned}
$$

where $S(N J)$ is a carleson cantor containing $\Phi^{-1}(S(I))$ and $J=\Phi^{-1}(I)$.

Since $\left|\mu_{1}(z)\right|^{2} /\left(1-|z|^{2}\right) d x d y$ and $\left|\mu_{2}(z)\right|^{2} /\left(1-|z|^{2}\right) d x d y$ are vanishing Carleson measures on $\mathbf{D}$, similar to proof of Theorem 3.1, we have

$$
\iint_{S(I)} \frac{|\eta(w)|^{2}}{1-|w|^{2}} d u d v=o(|I|), \quad|I| \rightarrow 0 .
$$


So $|\eta(w)|^{2} /\left(1-|w|^{2}\right) d x d y \in C M_{0}(\mathbf{D})$. It is obvious that $f \circ \Phi^{-1}$ is the quasiconformal extension of the normalized homeomorphism $h_{2} \circ\left(h_{1}\right)^{-1}$. Therefore, $h_{2} \circ\left(h_{1}\right)^{-1} \in T_{v}$ from Proposition 3.1 and $T_{v}$ is a subgroup of $T$.

\section{REFERENCES}

[1] K. Astala and F. W. Gehring, Injectivity, the BMO norm and the universal Teichmüller space, J. Anal. Math. 46 (1986), 16-57.

[2] K. Astala, T. Iwaniec and G. Martin, Elliptic partial differential equations and quasiconformal mappings in the plane, Princeton Uinv. Press, New Jersey, USA, 2009.

[ 3 ] K. Astala and M. Zinsmeister, Teichmüller space and BMOA, Math. Ann. 289 (1991), 613-625.

[4] A. Beurling and L. V. Ahlfors, The boundary correspondence under quasiconformal mappings, Acta. Math. 96 (1956), 125-142.

[5] C. Bishop AND P. Jones, Harmonic measure, $L^{2}$ estimates and the Schwarzian derivative, J. Anal. Math. 62 (1994), 77-113.

[6] M. D. Chen, D. G. Deng and R. L. Long, Real analysis, 2nd ed., Higher Education Press, Beijing, 2008 (in Chinese).

[7] G. CUI, Integrably asymptotic affine homeomorphisms of the circle and Teichmüller spaces, Sci. China, Ser. A. 43 (2000), 267-279.

[8] G. Cui and M. Zinsmeister, BMO Teichmüller space, Illinois J. Math. 48 (2004), 12231233.

[9] A. Douady and C. J. Earle, Conformally nature extension of homeomorphisms of the circle, Acta. Math. 157 (1986), 23-48.

[10] P. L. Duren, Theory of $H^{p}$ spaces, Academic Press, New York and London, 1970.

[11] C. J. Earle, V. Markovic and D. Saric, Barycentric extension and the Bers embedding for asymptotic Teichmüller space, Contemporary Math. 311 (2002), 87-105.

[12] F. P. GARDiner, Teichmüller theory and quadratic differentials, Wiley-Interscience, New York, 1987.

[13] F. P. GaRdiner and N. LAKIC, Quasiconformal Teichmüller theory, American Mathematical Society, New York, 2000.

[14] J. B. Garnett, Bounded analytic functions, Academic Press, New York, 1981.

[15] Y. Hu AND Y. SHEn, On quasisymmetric homeomorphisms, Israel J. Math. 191 (2012), 209-226.

[16] O. Lehto, Univalent functions and Teichmüller space, Springer-Verlag, New York, 1986.

[17] Z. LI, Quasiconformal mappings and Teichmüller space, Peking University Press, Beijing, 2013 (in Chinese).

[18] S. NAG, The complex analytic theory of Teichmüller space, Wiley-Interscience, 1988.

[19] Ch. Pommerenke, On univalent functions, Bloch functions and VMOA, Math. Ann. 123 (1978), 199-208.

[20] Ch. Pommerenke, Boundary behaviour of conformal maps, Springer-Verlag, Berlin, 1992.

[21] D. Sarason, Functions of vanishing mean oscillation, Trans. Amer. Math. Soc. 207 (1975), 391-405.

[22] Y. SHEN, Weil-Petersson Teichmüller space, arXiv:1304.3197v3.

[23] Y. Shen and H. Wei, Universal Teichmüller space and BMO, Adv. Math. 234 (2013), $129-148$.

[24] K. Strebel, Point shift differentials extremal quasiconformal mappings, Ann. Acad. Sci. Fenn. A. I. Math. 23 (1998), 475-494. 
Yan $\mathrm{Wu}$

LMiB and School of Mathematics and Systems Science BEIHANG UNIVERSITY

BEIJING, 100191

P.R. CHINA

SCHOOL OF SCIENCE

LINYI UNIVERSITY

SHANDONG, 276005

P.R. CHINA

E-mail: BY1209113@buaa.edu.cn wuyan@lyu.edu.cn

Yi Qi

LMIB and School of Mathematics and Systems Science BEIHANG UNIVERSITY

BEIJING, 100191

P.R. ChINA

E-mail: yiqi@buaa.edu.cn 